\title{
HRI and the Future of Law
}

\author{
Eduard Fosch Villaronga \\ Law, Governance and Technology Department \\ University of Twente \\ Enschede, The Netherlands \\ +31 (0)53 4899154 \\ e.foschvillaronga@utwente.nl
}

\author{
Michiel A. Heldeweg \\ Law, Governance and Technology Department \\ University of Twente \\ Enschede, The Netherlands \\ +31534896834 \\ m.a.heldeweg@utwente.nl
}

\begin{abstract}
Following Sinek's what-how-why model, this project is about the creation of a dynamic regulatory instrument that co-evolves with robot technology development (what), using a robot and a regulatory impact assessments and evaluation settings (simulation and living labs) for empirical testing (how), for three reasons (why): (1) to provide the robot users with a comprehensive protection (not only focused on technical matters); (2) to provide roboticists with a practical tool to know what regulations have to take into consideration into the life-cycle process of the robot; and (3) to fill the existing regulatory gaps caused by the fastness of the technological progress and avoid over-/under- regulated scenarios.
\end{abstract}

\section{INTRODUCTION}

Great expectations and major concerns accompany the development and possible uses of robotics in many areas of life and in many forms, such as drones and care robots. Possible pros and cons require careful regulatory attention, both as regards to technological aspects and with respect to societal/ethical appraisal [1], especially when it comes to the transition from the in silico and in vitro phases, i.e. design, creation of the robot; to the in vivo testing and the actual implementation/ of the robot.

The latter is relevant in respect of preserving constitutional rights and principles such as to/on safety/life, privacy (i.e. data protection), dignity and autonomy. As currently there is still a lack of interdisciplinary analysis on and assessment of the impact of robotics' technology on citizens/society, there is the need to develop not only responsible research initiatives [2], but also regulatory instruments that can co-evolve with the (robot) technology development, to provide both certainty to specific types/uses of (robot) technology, and inform new general (robotic) technology policies respectful to the afore constitutional principles and rights.

Seen robotics market exponential growth, this project focuses on the intertwinement between robotic technology and regulatory development. It aims at creating a dynamic regulatory instrument that can co-evolve with the advancement of robotic technology (see Fig. 1). The idea is to establish a methodology, using a robot impact assessment (ROBIA) and evaluation settings (simulation and living labs) for empirical testing, to study the pros and cons of this technology both at the technical and legal level. The model incorporates an ex-post Regulatory Impact Assessment (REGIA) to allow the incorporation of the findings into the legislative framework. This is consistent with the Responsible Research and

Permission to make digital or hard copies of part or all of this work for personal or classroom use is granted without fee provided that copies are not made or distributed for profit or commercial advantage and that copies bear this notice and the full citation on the first page. Copyrights for third-party components of this work must be honored. For all other uses, contact the Owner/Author

Copyright is held by the owner/author(s).

HRI '17 Companion, March 06-09, 2017, Vienna, Austria

ACM 978-1-4503-4885-0/17/03.

http://dx.doi.org/10.1145/3029798.3038313
Innovation (RRI) initiative, which aims at promoting the communication and involvement of all the stakeholders in research and innovation at a very early stage to obtain a common understanding of the impacts and outcomes of their actions to successfully balance outcomes and options in terms of societal needs and moral values and use these considerations as functional requirements for design and development of new technologies [2]. This model will provide the robot users with a type of comprehensive legal protection that goes beyond the mere physical safety-related protection given by current technical standards. At the same time, this will provide roboticists with a practical tool that will help them identify which regulations they have to take into consideration into the life-cycle process of the robot (design, creation, implementation). And, last but not least, the iteration of the model will help fill the existing regulatory gaps caused by the fastness of the technological progress preventing, at the same time, over- or under- regulated scenarios.

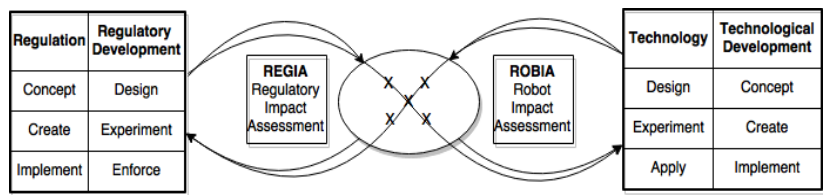

Figure 1. Dynamic Regulatory Model for Robotic Technologies

\section{THE MODEL}

A roboticist building a robot that interacts with humans may be clueless about what regulations apply to it, whether the robot behavior needs to be regulated by design [3] or whether $s /$ he is in charge of it. Sometimes, even the law is not prepared to accommodate new types of technology right away, e.g. driverless cars [4]. An instrument that could link robot development and legislation, therefore, could be of help to roboticists - an ROBIA in Fig. 1 [5], and ultimately to the users that could, as a result, enjoy safer technology. Some parts of the ROBIA, i.e. mainly the matching between the robot and the legislation that applies to it, could be automated as other initiatives have done for consumer products [6-7].

Impact assessments in the legal domain are currently seen merely as an accountability tool, i.e. a way to show that (in this case) a roboticist is compliant with the legal framework. This means that the mere fulfillment of the accountability requirement (through impact assessment) does not feed back into the legal system per se and, therefore, the law is not (easily) updated with the new advancements in technology - it is currently a separate instrument. A mechanism that could extract relevant knowledge from these accountability tools and apply it to the regulatory development - arrow from technology box to regulatory box, could help building grounded legal frameworks that could, later 
on, be applied to new projects. Depending on the nature of the interests involved, and the context or scale of the development among other variables, the regulatory choice balancing opportunities and threats will vary from criminal/civil sanctions to insurance conditions including others [8].

After having fed back into the regulation, ex-post legislative evaluations could help improve the system as long as the assessment is properly conducted and it has quality [9] - REGIA in Fig. 1. An example may ease the explanation. Tufts University is working with robotic therapy with non-neurotypical children [10]. In 2015, the therapy focused on young children. In 2017, the therapy will focus on young teenagers and in the future, they have plans to focus on teenagers and young adults. A legal assessment was provided in 2015 because it was not clear what framework should be applied to them [11]. The researchers have the intention to provide it for 2017. Further research will extract knowledge from both legal assessments and use it as a framework for following experiments - with young adults. The novelties, newly arisen issues, and modifications found in different age subjects will be included in the framework.

In order to both protect the user (a truly user-centered approach) and to extract empirical knowledge for regulatory purposes, some evaluation methods can be used in the robot development simulations and living labs:

a) The use of simulation for human-robot interaction (HRI) studies is very recent [12]. This type of simulators are much more cost-effective and personalized than the creation of a living lab, everything is recordable and reproducible, it offers concrete progress instead of abstract problems and it can predict the type of living lab needed for testing the physical prototype.

b) Originated in Japan, the use of living lab dates from 2003 [13]. They are conceptual regions or districts for experimental testing for robotic technologies that include experimental regulations for socio-economic revitalization and special regulatory measures, e.g. preferential treatment in taxation [4].

This connects with the regulatory system as this relates to various phases of the robot (use in) development, for instance in the case of the living lab, to allow testing of the robot (use) in development and to see if experimental regulations have been successful, to next upscale to nationwide regulations.

Within the regulatory system the choice of proper instruments requires navigation between prospective public law (e.g. permits), prospective private law (e.g. standards and insurance), retrospective public law (e.g. sanctions and regulatory recalls) and retrospective private law (e.g. liability) [8].

\section{CONCLUSIONS}

The need for overarching legal design analysis of the dynamics of regulation on robotics and the options for enhancing an open structured regulatory approach becomes evident in the light of the exponential growth of service robot technology that interact with humans.

Because of that, a dynamic regulatory model has been introduced in order to avoid an over- or under- regulated scenario that could frustrate both robotic development and user protection. This secures stakeholder-involvement in collective legal action toward effective, efficient and legitimate closure of possible future regulatory gaps.

\section{ACKNOWLEDGMENTS}

Our thanks to the Tech4People program of the University of Twente to financially support this research.

\section{REFERENCES}

[1] 2015/2103(INL) May, 2016. European Parliament Draft Report on Civil Law Rules on Robotics.

[2] DG For Research and Innovation Science in Society, 2013. Options for Strengthening Responsible Research and Innovation. European Commission

[3] Leenes, R., and Lucivero, F. 2014. Laws on robots, laws by robots, laws in robots: Regulating robot behaviour by design. Law, Innovation and Technology, 6(2), 193-220. DOI:10.5235/17579961.6.2.194

[4] Weng, Y. H., et al. 2015. Intersection of "Tokku" special zone, robots, and the law: a case study on legal impacts to humanoid robots. International Journal of Social Robotics, 7(5), 841-857. doi:10.1007/s12369-015-0287-x

[5] Fosch-Villaronga, E. 2015. Creation of a Care Robot Impact Assessment. WASET, International Journal of Social, Behavioral, Educational, Economic, Business and Industrial Engineering, 9(6), pp. 1867-1871

[6] Arkin, R. C., et al. 2009. An ethical governor for constraining lethal action in an autonomous system. No. GIT-GVU-09-02. Robot Lab, GeorgiaTech, Atlanta.

[7] United States Consumer Product Safety Commission Regulatory Robot. Available at: www.cpsc.gov/Business-Manufacturing/Regulatory-Robot/Safer-Products-Start-Here

[8] Smith, B. W. 2016. Regulation and the Risk of Inaction. In Autonomous Driving Maurer, M., J. et al. Ed. Springer, Berlin, 571-587.

[9] Mastenbroek, E. et al. 2016. Closing the regulatory cycle? A meta evaluation of ex-post legislative evaluations by the European Commission. Journal of European Public Policy, 23:9, 1329-1348.

[10] Data Analysis and Collection through Robotic Companions and LEGO Engineering with Children on the Autism Spectrum project. CEEO, Tufts University, US. See roboautism.k12engineering.com/?page_id $=2$

[11] Fosch Villaronga, E., and Albo-Canals, J. 2015. Boundaries in Play-based Cloud-companion-mediated Robotic Therapies: From Deception to Privacy Concerns. In Conference Proceedings New Friends. Heerink, M. et al. Ed. 164(6), 597-600.

[12] Dome project at Nishida Lab, 2015: www.ii.ist.i.kyotou.ac.jp/wordpress/wpcontent/uploads/2015/05/DomeDisplayDemoR3.pdf (in Japanese)

[13] Japanese Ministry of Economy, Trade and Industry (METI): www.meti.go.jp/english/policy/mono_info_service/robot_ind ustry 